\title{
Cryopreservation of pig embryos: adaptation of vitrification technology for embryo transfer
}

\author{
J. R. Dobrinsky \\ Germplasm and Gamete Physiology Laboratory, Agricultural Research Service, \\ US Department of Agriculture, Beltsville, MD 20705, USA
}

\begin{abstract}
Great advancements in cryopreservation of pig embryos have been made since the last International Conference on Pig Reproduction (ICPR). In 1997, there were standard methods to cryopreserve germplasm and embryos of most livestock species, except for the pig, and development of this technology for use in the international pig industry was slow and in the early stages. Since 1997, there have been advancements in cryopreservation of pig embryos, with reports of production of live offspring after transfer of frozen-thawed and vitrified-warmed pig embryos. This review summarizes the progress in cryopreservation of pig embryos since 1997. Cellular and molecular biology have been used to understand the hypothermic sensitivity of pig embryos. Development of delipation technology has provided the first evidence that intracellular lipids are linked to hypothermic sensitivity. Cytoskeletal stabilization and vitrification have led to the production of live offspring from vitrified-warmed and transferred embryos. Recently, technology has been developed for cryopreservation of pig morulae. Development of open pulled straws has provided more rapid rates of cooling during vitrification and has been effective for cryopreservation of pig embryos. Although improvements and refinements of the technologies will continue, it is now time for the pig industry to consider cryopreservation of pig embryos as a tool for pig production and for propagation of select herd genetics, while maintaining germplasm resources for the future.
\end{abstract}

\section{Introduction}

Conservation of genetic resources is essential for making desirable genes and germplasm available to meet the needs of the future. Banking of germplasm from animals with desirable genetic, production or disease resistance traits will facilitate acquisition and characterization of potentially useful germplasm, ensure genetic variation through preservation of selected stocks and facilitate the use of useful germplasm in research and industry in the future. Implementation of methodologies for long-term preservation and transfer of pig embryos would provide a foundation for effective use of the world's most valuable genetic resources, while modernizing production and enhancing genetic improvement programmes (Dobrinsky, 1997).

Disease transmission and other health concerns limit the transport of live animals and, hence, subsequent propagation globally. The use of embryos in addition to spermatozoa 
represents a major increase in the global efficiency of transmitting improved genetic potential. The ability to preserve maternal genetic material enables improvement of genetic potential in a form other than the live animal. The development of reliable techniques for preservation of pig embryos could not have been more timely for the pig industry, considering the most recent outbreaks of foot and mouth disease (FMD) in Europe and other parts of the world in February 2001 (Brownlie, 2001; N. C. Steele, personal communication). After starting in England, the infection had spread throughout the UK within a few weeks and cases were reported in the Netherlands, France and the Republic of Ireland. The most proven methodology for eradication of FMD is 'stamping out', which involves extermination of all animals, with no exceptions, from the ring perimeter inwards toward the site of infection, which denies the virus a host to support replication. Restriction of movement is integral to this strategy, as no movement of hosts or passive vectors (humans, equipment and product) beyond the ring radius is permitted. Pursuit of a stamping out policy implies that the long-term value of commodities for export is more valuable than the short-term losses incurred by mass slaughter. Attempts to control and eradicate the epidemic resulted in the slaughter of over 4 million head of livestock in the UK alone, while FMD outbreaks in other parts of the world (for example, Taiwan in the mid-1990s) and attempts to control and eradicate the disease have caused the unintentional loss of germplasm resources worldwide (Brownlie, 2001; N. C. Steele, personal communication). If embryos had been cryopreserved and held in long-term storage, permanent germplasm losses could have been averted, as present lines could be regenerated through embryo transfer once the threat of the disease outbreak has subsided. Herd repopulation by genetic rescue from cryopreservation and embryo transfer of stored genetic lines, as well as import of genetically superior embryos for transfer, would enhance genetic repopulation.

The development of a repeatable method for the long-term preservation of pig embryos, coupled with embryo transfer, would provide numerous applications for production, research and medicine, including indefinite embryo storage, line regeneration, line proliferation, increased selection pressure in select herds, rescue of premium genetics from diseased herds, international export and import of potential breeding stock, and insurance for select genetic lines that are critical for the advancement of animal production and biotechnology. Furthermore, the global transport of genetically superior embryos to developing countries will enhance indigenous pig populations greatly to meet the needs of a growing world population. The conservation and preservation of pig genetics would enhance the improvement and usage of animal production techniques such as sperm sexing, artificial insemination, in vitro fertilization and development of non-surgical transfer of pig embryos. Collectively, these technologies would have a major impact on pig production worldwide (Dobrinsky et al., 2000).

Early research on cryopreservation of pig embryos has been reviewed in great detail (Dobrinsky, 1997). Since then, new technology has been developed to cryopreserve early hatched blastocyst stage pig embryos, which has resulted in the world's first live piglets born after cryopreservation by vitrification. This technology was an interim solution suitable only for local movement of embryos within the country of origin; international rules on embryo transport outside the country of origin mean that only aseptic embryos with an intact zona pellucida can be exported. In this review, the most recent developments in pig embryo cryopreservation technology are summarized. The methodology for cryopreservation of morula stage pig embryos is described, including adaptation of delipation for cryopreservation of embryos in the absence of much of their intracellular lipids. Furthermore, the USDA swine embryo cryopreservation technology (USECT), a non-invasive methodology designed to cryopreserve zona pellucida intact, non-micromanipulated, pre-blastocyst stage 
pig embryos, is described. Data on development of live offspring after transfer of delipatedcryopreserved embryos and USECT embryos are also presented.

\section{Cryopreservation of pig embryos}

\section{Cytoskeletal stabilization}

Dobrinsky (1997) reported ongoing pregnancies after transfer of cytoskeletal-stabilized, vitrified hatched blastocysts. Dobrinsky $(1996,1997)$ and Dobrinsky et al. (2000) reported cellular disruption, specifically to the embryonic cytoskeleton, during and after cryopreservation, for developing methodologies to circumvent such disruptions that would lead to the production of live offspring after cryopreservation and subsequent embryo transfer. Microfilament damage during vitrification of pig embryos and the use of cytochalasins before and during cryopreservation were assessed to help prevent damage and to stabilize the plasma membrane. Our hypothesis was based on the observation that cryoprotectants and cryopreservation disrupted the embryonic cytoskeleton, specifically filamentous actin. If the actin microfilaments were dismantled reversibly (or depolymerized) before cryopreservation, they might reform (or repolymerize) normally after rehydration. When embryos were treated and vitrified under the influence of a depolymerizing agent (cytochalasin b), after rehydration the filamentous actin repolymerized normally in the absence of cytochalasin b and embryos resumed development in vitro (Dobrinsky et al., 2000). Morulae and early blastocysts did not survive cryopreservation with or without cytochalasin b (Table 1). Expanded and hatched blastocysts showed low rates of survival after vitrification (22-29\%). However, vitrification under the influence of cytochalasin b improved the survival of expanded and early hatched blastocysts significantly. Although cytochalasin b-treated expanded blastocysts had improved viability after vitrification $(60 \%)$, their development was still lower than that of early hatched blastocysts $(90 \%)$. Cytochalasin b did not improve development of expanded hatched blastocysts after vitrification ( $41 \%)$.

Trans-oviductal uterine catheterization was used for transfer of cytoskeletal-stabilized and vitrified embryos to seven surrogate females (29-33 embryos per female; Table 2). Two surrogate females were examined on day 25 of presumptive gestation; one recipient had four normal fetuses. Of the other five surrogate females, two farrowed five live and normal offspring each. These piglets were normal in appearance and had birth weights within the normal range for these hybrids. The surrogate mothers weaned eight of the ten offspring, which grew normally as assessed by growth rates and phenotypes, and no anatomical abnormalities were observed by macroscopic examination after weaning at day 28 after birth. Of these mature offspring, two boars and two gilts were retained for breeding and have successfully proven their fecundity, as the boars each inseminated three gilts, and the two gilt offspring were mated. The females farrowed normal litters.

In a second cryopreservation-embryo transfer trial (Table 3), all embryos were cultured for 3-5 $\mathrm{h}$ after warming and recovery. Only morphologically excellent to good recavitating embryos were transferred by trans-oviductal uterine catheterization with surgical embryo transfer. Of seven recipient surrogate females, four farrowed a total of 29 live offspring, with litter sizes of 10, 10,6 and 3. Select offspring from these litters have been raised to maturity to study their fecundity. At least three generations of normal pigs have been produced from this founder line of offspring produced from cryopreserved embryos. Stabilized vitrification is a viable method for the long-term preservation of pig embryos. This work enabled the repeatable production of live offspring after transfer of cytoskeletal-stabilized and vitrifiedwarmed embryos into surrogate females. 
Table 1. Development of morphologically different stages of pig embryos after cryopreservation by vitrification under the influence of microfilament stabilizer cytochalasin b

\begin{tabular}{|c|c|c|c|c|c|c|}
\hline \multirow[b]{2}{*}{ Stage of development } & \multicolumn{3}{|c|}{ Vitrification control } & \multicolumn{3}{|c|}{ Vitrification + cytochalasin b } \\
\hline & $n$ & Development & $\%$ Development & $n$ & Development & $\%$ Development \\
\hline Morula or blastocyst & 17 & 0 & $0^{a}$ & 17 & 1 & $6^{a}$ \\
\hline Expanded blastocyst & 27 & 6 & $22^{a b}$ & 25 & 15 & $60^{b x}$ \\
\hline Hatched blastocyst & 1 & & & & & \\
\hline $\begin{array}{l}<400 \mu \mathrm{m} \text { in diameter } \\
\text { Hatched blastocyst }\end{array}$ & 36 & 10 & $28^{\mathrm{b}}$ & 48 & 43 & $90^{c x}$ \\
\hline$>400 \mu \mathrm{m}$ in diameter & 14 & 4 & $29^{a b}$ & 22 & 9 & $41^{b}$ \\
\hline
\end{tabular}

a-c Values with different superscripts within columns are significantly different $(P<0.05 ;$ ANOVA-GL.M).

${ }^{x}$ Value with superscript is significantly different from control value within the row $(P<0.01$; chi-squared analysis $)$.

Data are írom Dobrinsky et al. (2000).

Table 2. Development of cytoskeletal-stabilized and vitrified hatched blastocyst stage pig embryos after embryo transfer

\begin{tabular}{lccl}
\hline Recipient & $\begin{array}{c}\text { Number of embryos } \\
\text { transferred }\end{array}$ & Embryo quality* & Embryo development \\
\hline $\mathrm{H}$ & 33 & 16 & 5 offspring \\
$\mathrm{I}$ & 33 & 17 & None \\
$\mathrm{J}$ & 32 & 19 & 5 offspring \\
$\mathrm{K}$ & 29 & 17 & None \\
$\mathrm{L}$ & 30 & 12 & None \\
Total & 157 & $81(52 \%)$ & 10 offspring $(n=5.0$ \\
& & & average litter size $)$ \\
\hline
\end{tabular}

*Number of excellent to good quality embryos at the time of transfer, 3-5 h after rehydration.

Data are from Dobrinsky et al. (2000).

\section{Vitrification research}

Kuwayama et al. (1997) reported that, of a range of pig embryos from the morulae to the hatched blastocyst stage, blastocysts achieved the highest survival rates in vitro with the vitrification treatments described. Much of the earlier reviewed literature (Dobrinsky, 1997) supports the contention that peri-hatching blastocysts are able to survive some forms of cryopreservation. Vajta et al. (1997) introduced 'open pulled straw' (OPS) vitrification. This methodology, which is based on a decreased diameter of straws that subsequently increases surface area to volume ratio, is designed to increase the rate of cooling of solutions during vitrification. Vajta et al. (1997) showed high rates of embryo development from morulae and blastocysts to hatched blastocyst stages (70-90\%) in vitro. Unfortunately, at the time of these experiments, the authors did not transfer embryos to study the developmental competence of OPS-vitrified embryos. Holm et al. (1999) also reported high rates of survival in vitro after OPS vitrification of 346 pig blastocysts. After warming, the embryos were cultured for 24 or $48 \mathrm{~h}$ or transferred surgically to synchronized recipients. In vitro, $88 \%$ and $71 \%$ of warmed blastocysts were reported to re-expand or hatch in culture, respectively. After embryo transfer, no recipients farrowed. 
Table 3. Development of cytoskeletal-stabilized and vitrified hatched blastocyst stage pig embryos after embryo transíer

\begin{tabular}{lccl}
\hline Recipient & $\begin{array}{c}\text { Number of embryos } \\
\text { transferred }\end{array}$ & Embryo quality* & Embryo development \\
\hline$M$ & 32 & 26 & 10 off́spring \\
$N$ & 33 & 27 & None \\
$O$ & 31 & 20 & None \\
$P$ & 31 & 19 & 10 offspring \\
$Q$ & 32 & 20 & 6 offspring \\
$R$ & 33 & 16 & None \\
S & 32 & 15 & 3 offspring \\
Total & 224 & $143(64 \%)$ & 29 offspring $(n=7.25$ \\
& & & average litter size) \\
\hline
\end{tabular}

"Number of excellent to good quality embryos at the time of transfer, at 3-5 h aiter rehydration; remainder of embryos transferred were marginally developing, showing some cellular disruption but maintaining recavitation.

Data are from Dobrinsky et al. (2000).

Berthelot et al. (2000) reported the first live offspring after OPS vitrification of pig embryos. Morulae and unhatched blastocysts from Large White hyperprolific (LWh) and Meishan gilts were used to test the OPS vitrification method with two media. The viability of vitrifiedwarmed embryos was estimated by the percentage of embryos that developed to the hatched blastocyst stage in vitro or by birth after transfer. In the first experiment, two cryoprotectant dilution media were compared for cryopreservation of Meishan and LWh blastocysts: the culture medium was a standard Hepes-buffered TCM $199+20 \%$ new born calf serum (NBCS) medium and PBS was a PBS $+20 \%$ NBCS medium. After a two-step equilibration in ethylene glycol, dimethyl sulphoxide and sucrose, 2-5 blastocysts were loaded into OPS and plunged into liquid nitrogen: The embryos were warmed and a four-step dilution with decreasing concentrations of sucrose was applied. In PBS, LWh blastocysts $(27 \%)$ had a lower viability in vitro than did Meishan blastocysts $(67 \% ; P=0.001)$. No significant difference was observed between genotypes ( $41 \%$ for LWh and $43 \%$ for Meishan blastocysts) in TCM and both viability rates were lower than that of the control group. In the second experiment, morula stage LWh and Meishan embryos were vitrified and warmed using PBS. The viability rate was low and was not significantly different between LWh $(11 \%)$ and Meishan (14\%) embryos. In the third experiment, 200 Meishan and $200 \mathrm{LWh}$ blastocysts were vitrified-warmed as described in the first experiment (PBS). Twenty embryos were transferred to each of 20 Meishan recipients. The farrowing rate was $55 \%$ and recipients farrowed four and five piglets (median) for Meishan and LWh blastocysts, respectively. In this experimental design, the OPS vitrification method was appropriate for cryopreservation of unhatched pig blastocysts.

\section{Embryo delipation}

The emphasis in our laboratory has been on cryopreservation of morula or early blastocyst stage pig embryos. Pig embryos suffer from severe sensitivity to hypothermia, which limits their ability to withstand conventional cryopreservation. Research has focused on the high lipid content of pig embryos and its role on hypothermic sensitivity and cryosurvival. Our previous data indicate, as do most reports in the literature, that morula stage pig embryos have little or no survival after cryopreservation (Dobrinsky, 1997). Nagashima et al. (1995) 
provided the first real evidence that intracellular lipid was associated with cooling or cryosensitivity of pig embryos. These workers isolated lipid from the embryo by centrifugation, used micromanipulation to remove (or suck) lipid from the embryo (delipation) and cooled or cryopreserved two- to eight-cell pig embryos. More than half of the delipated embryos survived cryopreservation, whether they had been frozen immediately after delipation or after further culture (to determine mitotic competence) before freezing, whereas none of the control embryos survived. Normal piglets were obtained from at least one recipient after unfrozen delipated and frozen delipated embryos were transferred. It is clear that pig embryos gain some form of tolerance to chilling when their lipid content is reduced. This was the first direct evidence that early cleavage stage embryos can survive cryopreservation after delipation and that the loss of cytoplasmic lipid is compensated for later in development. These observations by Nagashima et al. (1995) are a landmark in understanding the sensitivity of pig embryos to cooling and cryopreservation.

Dobrinsky et al. (1999) aimed to determine whether morulae and early blastocysts could survive delipation and cryopreservation. Embryos were delipated and frozen by conventional freezing using procedures described by Nagashima et al. (1995); delipated-vitrified embryos were cryopreserved with cytoskeletal stabilization throughout micromanipulation and vitrification. Control and delipated non-cryopreserved embryos developed at high rates in vitro. After cryopreservation, $89 \%$ of delipated-frozen embryos and $83 \%$ of delipatedvitrified embryos underwent advanced embryonic development in culture for $48 \mathrm{~h}$, including cavitation and blastocyst expansion. After cryopreservation and subsequent embryo transfer, three of seven recipients that received delipated-frozen embryos delivered a total of 14 live piglets, and three of four recipients that received delipated-vitrified embryos delivered a total of 13 live piglets. These studies confirm that morulae and early blastocyst stage pig embryos can withstand cryopreservation by conventional freezing or vitrification after removal of their cytoplasmic lipid and remain developmentally competent after transfer.

Although these results indicate that intracellular lipid is involved directly in hypothermic sensitivity of morulae and early blastocyst stage embryos, delipation disrupts the zona pellucida of the embryos. It is a requirement that embryos to be transported internationally must have an intact zona pellucida (Stringfellow and Seidel, 1998). Dobrinsky et al. (2001) aimed to develop non-invasive methodology for cryopreservation of non-micromanipulated, zona pellucida intact morula and early blastocyst stage pig embryos to enable long-term preservation and future global transfer of uterine stage pig embryos.

\section{Non-invasive embryo vitrification}

In a preliminary study, we cryopreserved centrifuged, intact morulae and early blastocyst stage embryos (Dobrinsky et al., 2001). Embryos were either centrifuged to isolate lipid or not centrifuged (intact whole embryos), and cryopreserved by conventional freezing or vitrification. Embryos were recovered from cryopreservation and cultured for $48 \mathrm{~h}$. Noncentrifuged frozen and non-centrifuged vitrified morulae and early blastocyst stage embryos did not develop in vitro. Centrifuged frozen-thawed and centrifuged vitrified-warmed embryos showed 60-70\% survival in vitro after cryopreservation, and most developed a morphologically acceptable blastocoel after $48 \mathrm{~h}$ of culture. However, embryos that developed to early blastocysts did not continue to develop or hatch in vitro, which is typical for control embryos cultured for $48 \mathrm{~h}$. Blastocysts that developed did not expand and the zona pellucida did not undergo any thinning, which was typical for culture control embryos. Vital staining by propidium iodide exclusion indicated that most embryos with a blastocoel were alive and intact, but their development appeared to be arrested, as they did not undergo any further cell 
Table 4. Effect of centrifugation before cryopreservation and development in vitro $(72 \mathrm{~h})$ of control and cryopreserved morula and early blastocyst stage pig embryos after zona pellucida removal

\begin{tabular}{lcccc}
\hline $\begin{array}{l}\text { Type of } \\
\text { cryopreservation }\end{array}$ & Centrifugation & $\begin{array}{c}\text { Number of } \\
\text { embryos cultured }\end{array}$ & $\begin{array}{c}\text { Number of embryos } \\
\text { that developed (\%) }\end{array}$ & $\begin{array}{c}\text { Number of cells } \\
\text { in blastocysts }\end{array}$ \\
\hline $\begin{array}{l}\text { Frozen } \\
\text { Frozen }\end{array}$ & + & 25 & $24(96)^{\mathrm{a}}$ & $100.3 \pm 16.2^{\mathrm{a}}$ \\
Vitrified & - & 22 & $4(18)^{\mathrm{b}}$ & $64.3 \pm 17.2^{\mathrm{b}}$ \\
Vitrified & + & 25 & $21(84)^{\mathrm{a}}$ & $115.4 \pm 140^{\mathrm{a}}$ \\
None & - & 26 & $2(8)^{\mathrm{b}}$ & $60.0 \pm 4.0^{\mathrm{b}}$ \\
& + & 20 & $20(100)^{\mathrm{a}}$ & $216.6 \pm 13.4^{\mathrm{c}}$ \\
\hline
\end{tabular}

a-cValues within columns with different superscripts are significantly different $(P<0.05)$.

Data are from Dobrinsky et al. (1999).

division or morphological progression of development in culture. It was then investigated whether centrifuged cryopreserved morulae and early blastocyst stage embryos can continue to develop normally in vivo. Centrifuged-vitrified embryos were warmed and transferred to recipient females, but pregnancies were not established. Furthermore, embryos could not be recovered from three recipients at slaughter $72 \mathrm{~h}$ after embryo transfer. No embryos were found when uterine flushings from the recipients were examined. Lipid isolated but undelipated, zona pellucida intact embryos cease to develop in vitro and did not develop in vivo. It is possible that embryo lysis might result from a local cytotoxic effect of the isolated lipid remaining inside the intact zona pellucida. In centrifuged, cryopreserved morulae and early blastocyst stage embryos either the embryo itself or the zona pellucida is altered in a way that prevents further development. However, the surviving embryos did develop a morphologically acceptable blastocoel before they became dormant.

Embryos were cryopreserved by conventional freezing or vitrification, with or without centrifugation (Dobrinsky et al., 2001), and after indefinite storage in liquid nitrogen the embryos were warmed and processed through dilution and rehydration. After rehydration, the zona pellucida was removed and zona-free embryos were placed into culture medium to monitor development after thawing. Centrifuged, non-cryopreserved, zona pellucida-removed embryos served as a culture control group. As expected, non-centrifuged, cryopreserved morulae and early blastocyst stage embryos with their zona pellucida removed did not develop in vitro (Table 4). However, centrifuged, cryopreserved morulae and early blastocyst stage embryos with zona pellucida removed developed at high rates into blastocysts after $72 \mathrm{~h}$ in culture and, morphologically, resembled expanding hatched blastocysts. Furthermore, the type of cryopreservation had no effect on embryo survival in embryos with zonae pellucidae removed. Interestingly, centrifuged, non-cryopreserved, control embryos with zonae pellucidae removed, recovered from the same embryo donors and distributed randomly to treatment or control groups, also developed well in culture and undenwent a two-fold increase in number of cells after $72 \mathrm{~h}$ of culture compared with centrifuged, cryopreserved embryos with zonae pellucidae removed. Recovery from the entire cryopreservation procedure did slow down the rate of cryopreserved embryo development in vitro, as indicated by reduced cell numbers; however, the type of cryopreservation had no effect on mitotic activity in culture after zona pellucida removal.

Centrifuged, cryopreserved morulae and early blastocyst stage embryos were transferred to asynchronous surrogate females to determine whether this reduction in number of cells would affect developmental competence (Dobrinsky et al., 2001). As type of cryopreservation had no effect on in vitro embryo development to blastocysts and number of blastocyst cells, vitrification was selected as the method of cryopreservation as it is a simple and rapid 
Table 5. Development of centrifuged, cytoskeletal-stabilized and vitrified morulae and early blastocyst stage pig embryos after zona pellucida removal and embryo transfer

\begin{tabular}{llcc}
\hline & & \multicolumn{2}{c}{ Litter size } \\
\cline { 3 - 4 } Recipient & Status & Live offspring & Total offspring \\
\hline A & Not pregnant & - & - \\
B & Pregnant & 7 & 8 \\
C & Pregnant & 4 & 7 \\
D & Pregnant & - & 5 \\
E & Not pregnant & 9 & -10 \\
F & Pregnant & 7 & 7 \\
H & Pregnant & 7 & 8 \\
J & Pregnant & 4 & 5 \\
K & Pregnant & 8 & 8 \\
Total & Pregnant & 7 & 7 \\
\hline
\end{tabular}

Trial 1: recipients A-D; trial 2: recipients E-K.

Data are from Dobrinsky et al. (2001).

procedure for cryopreservation of large numbers of embryos destined for transfer in a short period of time. Large numbers of embryo donors underwent oestrous synchronization and superovulation, and embryos were recovered in bulk and cryopreserved using the new USDA swine embryo cryopreservation technology. All centrifuged and vitrified embryos were cryopreserved in bulk and stored in liquid nitrogen for 3-6 months. Naturally cyclic gilts at day 4 after oestrus (oestrus $=$ day 0 ), with at least two previous normal oestrous cycles, served as asynchronous $(-24$ to $-36 \mathrm{~h})$ recipients. In the first trial, centrifuged and vitrified embryos were warmed, rehydrated and their zona pellucida was removed and transferred (Table 5); three of four gilts farrowed eight, seven and four live healthy offspring. In the second trial, six of seven gilts farrowed a total of 42 offspring. Total recipient farrowing rate was $82 \%(9 / 11)$ with 61 live piglets born, averaging seven pigs per litter.

Select male and female offspring from these litters were retained on our farm and raised to sexual maturity (Dobrinsky et al., 2001). Their growth rates to day 165 of age were not different from the farm average. Three centrifuged and vitrified female offspring entered puberty normally and maintained normal oestrous cycles before mating. The gilts underwent normal pregnancies and each had a litter of piglets that grew normally and established normal fecundity. Two boars that developed from centrifuged and vitrified embryos entered puberty normally. One boar was trained for semen collection and was placed in our boar facility. Semen was collected from this boar, processed and extended for use in artificial insemination of three hybrid gilts in oestrus. These gilts became pregnant and had litters of piglets that grew normally and established normal fecundity. The other boar was placed on the farm and was used in natural mating of three hybrid gilts in oestrus. These gilts also became pregnant and had litters of piglets that grew normally and established normal fecundity.

\section{Conclusion}

In the conclusion of my last paper at ICPR, I began with the remarks '... Methods for preservation of pig embryos are in early stages of development, as are other technologies in 
pigs, such as non-surgical embryo transfer ( $\mathrm{Li}$ et al., 1996), that could make embryo cryopreservation practical and usable by the producer. Technologies available for embryo transfer in cattle, such as non-surgical embryo recovery and in vitro embryo production, are not readily available to the pig industry or are in early stages of development, making it impractical for the producer to use these biotechnical advances in swine. (Dobrinsky, 1997). In 2001, this is no longer the case. In our laboratory and others, vitrification technologies have been used to overcome the cooling sensitivity of pig embryos and methodologies have been developed that can produce high rates of live offspring after cryopreservation and embryo transfer. Although delipated morulae and early blastocysts can survive cryopreservation and maintain developmental competence to live offspring, the USDA swine embryo cryopreservation technology provides a non-invasive methodology to cryopreserve pig embryos at all stages of preimplantation, from zygotes to hatched blastocysts, and can produce live, healthy piglets that grow normally and are of excellent fecundity when mature. The in vivo development of cryopreserved embryos after transfer could be improved, as in most reports $<30 \%$ of transferred embryos actually develop to live offspring. Furthermore, a better understanding of the physiology, endocrinology and synchrony of embryo recipient (surrogate) females at the time of embryo transfer is required.

Implementation of methodologies for long-term embryo preservation and transfer in pigs would provide a foundation for effective use of the world's most valuable genetic resources on a global basis while modernizing production and enhancing genetic improvement programmes. It is now time for breeders and producers to adopt cryopreservation and transfer of pig embryos into pig production for propagating select genetic traits and maintaining germplasm resources for the future. Collectively, these technologies will have a major impact on pig production worldwide.

\section{References}

Berthelot F, Martinat-Botte F, Locatelli A, Perreau C and Terqui $M$ (2000) Piglets born after vitrification of embryos using the open pulled straw method Cryobiology 41 116-124

Brownlie I (2001) Strategic decisions to evaluate before implementing a vaccine programme in the face of a foot-and-mouth disease (FMD) outbreak British Cattle Veterinary Association Bulletin 22 March 1-5

Dobrinsky IR (1996) Cellular approach to cryopreservation of embryos Theriogenology 45 17-26

Dobrinsky IR (1997) Cryopreservation of pig embryos Journal of Reproduction and Fertility 52 301-312

Dobrinsky JR, Nagashima H, Pursel VG, Long CR and Johnson LA (1999) Cryopreservation of swine embryos with reduced lipid content Theriogenology 51164 (Abstract)

Dobrinsky JR, Pursel VG, Long CR and Johnson LA (2000) Birth of piglets after transfer of embryos cryopreserved by cytoskeletal stabilization and vitrification Biology of Reproduction 62 564-570

Dobrinsky JR, Nagashima H, Pursel VG, Schreier LL and Johnson LA (2001) Cryopreservation of morula and early blastocyst stage swine embryos: birth of litters after embryo transfers Theriogenology 55303 (Abstract)

Holm P, Vajta G, Machaty Z, Schmidt M, Prather RS, Greve $T$ and Callesen H (1999) Open pulled straw (OPS) vitrification of porcine blastocysts: simple procedure yielding excellent in vitro survival but so far no piglets following transfer Cryo-Letters $20307-310$

Kuwayama M, Holm P, Jacobsen $\mathbf{H}$, Greve $\mathbf{T}$ and Callesen $\mathbf{H}$ (1997) Successful cryopreservation of porcine embryos by vitrification Veterinary Record 141365

Nagashima H, Kashiwazaki N, Ashman RJ, Grupen CG and Nottle MB (1995) Cryopreservalion of porcine embryos Nature 374416

Stringfellow DA and Seidel SM (1998) Ethical and sanitary recovery, handling, preservation, movement and transfer of embryos. In Manual of the International Embryo Transfer Society pp 79-84. International Embryo Transfer Society, Savoy, Illinois

Vajta G, Holm P, Greve T and Callesen $H$ (1997) Vitrification of porcine embryos using the open pulled straw (OPS) method Veterinaria Scandinavica 38 349-352 